\title{
Seasonal antimicrobial activity of the volatile exometabolites of some tropical lianas during introduction
}

\author{
Evgeny Savitsky ${ }^{1 *}$, Tatyana Fershalova ${ }^{2}$ and Natalya Tsybulya $^{2}$ \\ ${ }^{1}$ Ural Branch: Institute Botanic Garden of the Russian Academy of Sciences (RASUBIBG), \\ Ekaterinburg 620144, Russia \\ ${ }^{2}$ Central Siberian Botanical Garden of the Siberian Branch of the Russian Academy of Sciences \\ (CSBG SB RAS), Novosibirsk 630090, Russia
}

\begin{abstract}
The dynamics of the seasonal activity of exometabolites was studied for the lianas from the families: Araceae - Epipremnum aureum G.S. Bunting, Monstera deliciosa Liebm. var. borsigiana и Araliaceae Hedera helix L. and Schefflera octophylla (Lour.) Harms. The antimicrobial activity of intact plants was evaluated by treating inoculated microbial test cultures Staphylococcus epidermidis, Escherichia coli and Candida albicans. in Petri dished with the volatile emissions of plants. Antimicrobial action was measured in comparison with the reference using the scale of phytoncide activity. The species and seasonal specificity of antimicrobial activity of exometabolites of intact plant leaves was revealed. All the studied plants exhibited moderate seasonal activity against $S$. epidermidis. Long-term seasonal activity was detected for $S$. octophylla. Pronounced activity against $E$. coli was detected in the species $H$. helix, $M$. deliciosa var. borsigiana and $S$. octophylla, while antifungal action was detected in the species E. aureum and S. octophylla. Substantial antifungal and antibacterial activity against $S$. epidermidis was observed during intense growth during summer and in the autumn-winter period. These experimental data provide evidence of species-specific antimicrobial activity during different periods of seasonal development of plants against the test objects.
\end{abstract}

\section{Introduction}

Some lianas growing under natural conditions in the forest regions of humid tropics and subtropics in south-eastern Asia, on the islands of the Pacific ocean and in Australia were chosen for experiment.

Under the conditions of introduction in greenhouses and indoors in the Urals and West Siberia, these taxons do not enter the generative phase but vegetate with high intensity. One of the major advantages of lianas is their ergonomics: they produce the green mass of

* Corresponding author: savicckiy@mail.ru 
leaves several times more than trees and bushes do, but lianas occupy a 2-3 times smaller area. The annual growth of the shoots may reach 2 to $4 \mathrm{~m}$ and even more, which exceeds the annual growth of trees and bushed by a factor of 5-10 [1]. As a rule, lianas are used in indoor planting to make a green wall phytocomposition, which may separate the space into functional zones. Keeping the optimal relation between the leaf area and room volume, it is possible to solve one of the most important modern problems, namely creation of healthy conditions for human life, in particular due to a decrease in the microbial contamination of indoor air with the help of intact plants. In the world practice, the antimicrobial activity of volatile emissions of Epipremnum pinnatum was proven against Staphylococcus aureus, $S$. saprophyticus [2]; the activity of the extracts of E. pinnatum [3] and Schefflera stellate [4] against some microbial test objects was established. Investigation of the dynamics of seasonal activity of exometabolites, for the lianas chosen for experiment, will allow us to select the assortment of plants for making phyto modules with continuous phytoncide action all year round.

\section{Material and methods}

Inoculated microbial test cultures of gram-positive bacteria Staphylococcus epidermidis (ATCC 25923), gram-negative bacteria Escherichia coli (ATCC 25922), and yeast-like fungi Candida albicans (ATCC 10231) were treated with the volatile compounds released from the intact leaves. The microorganisms were inoculated in Petri dishes on the surface of GRM agar medium for microorganism cultivation, Daily cultures of three test microorganisms were plated with the help of a microbiological loop $2 \mathrm{~mm}$ in diameter (streak inoculation) into the upper dish separated into three sectors. The lower dish contained freshly collected plant leaves distributed uniformly. The contact of the leaves with the medium was excluded. Each plant sample was tested three times.

Leaves for the study were collected from the same plants during different periods of their seasonal development: during intense and moderate growth of shoots and leaves. Experimental treatment of the inoculated microorganisms with the volatile emissions of plants was carried out from 12 to 16 p.m. in daytime at room temperature, and then the reference and experimental Petri dishes were placed into the thermostat for 18-20 hours at a temperature of $37^{\circ} \mathrm{C}$. Antimicrobial action of the volatile compounds was evaluated by comparing with the reference over the scale of phytoncide activity: 0 meant the absence of effect (a continuous growth of microbes in the culture), 1 point meant weak action (suppression of culture growth in the middle by not more than $20-30 \%$ of streak length); 2 points - moderate effect (suppression of the growth of culture up to $40-50 \%$ of streak length); 3 points — strong effect (insignificant growth of culture at the ends of streak); 4 points - very string effect (almost complete absence of the growth of microbial culture) [5].

Taxon ranking over their antimicrobial activity was carried out taking into account the average number of points during the vegetation period with respect to each test object and to all test objects using the scale: 0.4-1.4 points - low activity, 1.5-2.0 points - moderate activity, 2.1- 2.5 points - high activity, 2.6 points and more - very high activity. Activity ranked as 1.5 points and more was referred to as pronounced.

\section{Results}

Analysis of the obtained experimental data allowed us to reveal that the studied plants are characterized by seasonal variations of the parameter under investigation. Average points over the vegetation period show that three representatives of the studied plants exhibit moderate activity to all test objects (Fig. 1). 


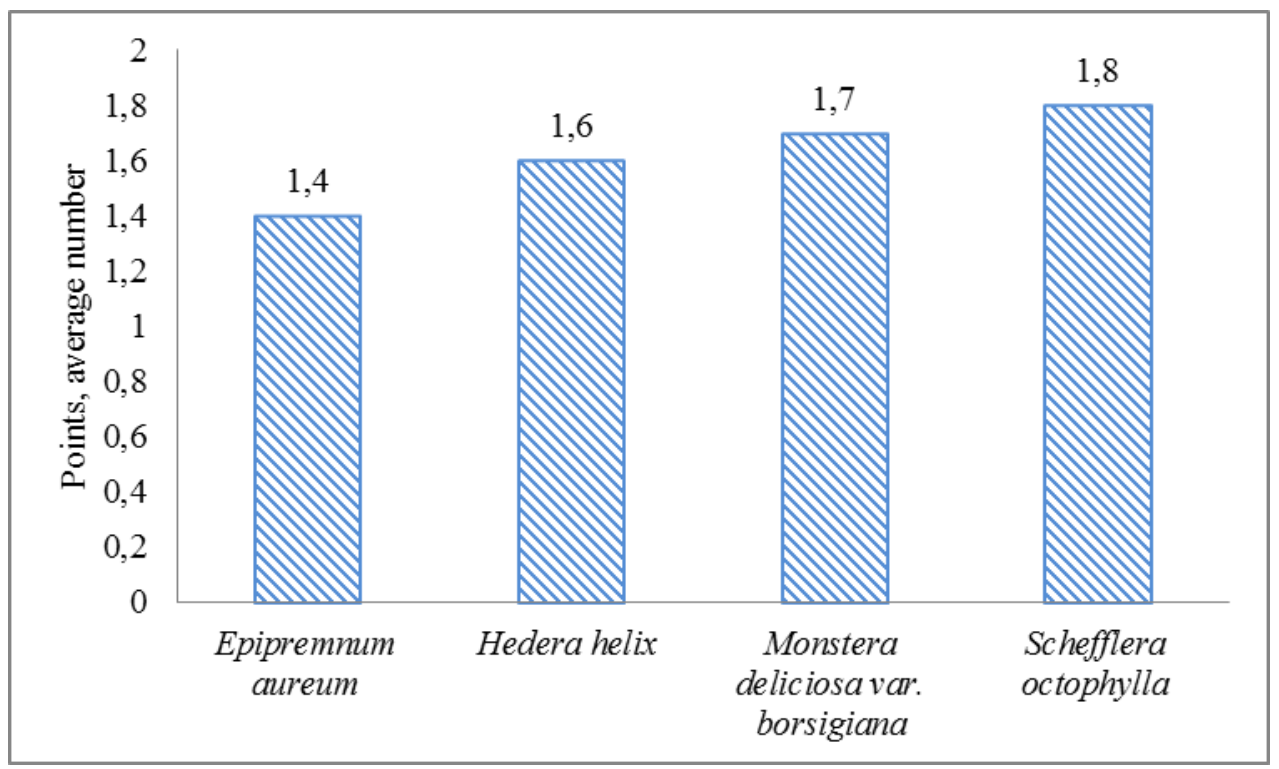

Fig. 1. Antimicrobial activity of the studied plant species to all test objects during the vegetation period (average number of points).

Pronounced bactericidal and fungicidal activity during the vegetation period (1.6-1.8 points) against all test objects was determined for $M$. deliciosa var. borsigiana, $S$. octophylla and H. helix .

Specificity and evaluation of the average seasonal activity of plants against microbial test objects are presented in Table 1.

Table 1. Comparison of the average seasonal activity of species and varieties of plants against microbial test objects

\begin{tabular}{|c|c|c|c|}
\hline \multirow{2}{*}{ Species, varieties } & \multicolumn{3}{|c|}{$\begin{array}{c}\text { Antimicrobial activity, average number of } \\
\text { points per season }\end{array}$} \\
\cline { 2 - 4 } & $\begin{array}{c}\text { S. } \\
\text { epidermidis }\end{array}$ & E. coli & C. albicans \\
\hline E. aureum & 1.5 & 1.0 & 1.7 \\
\hline $\begin{array}{c}\text { M. deliciosa } \text { var. } \\
\text { borsigiana }\end{array}$ & 2.0 & 1.8 & 1.2 \\
\hline H. helix & 1.7 & 1.8 & 1.3 \\
\hline $\begin{array}{c}\text { S. octophylla } \\
\text { Activity against test } \\
\text { objects }\end{array}$ & 2.0 & 1.6 & 1.5 \\
\hline
\end{tabular}

All the studied plant taxons exhibited moderate effect against $S$. epidermidis. Pronounced effect against $E$. coli was demonstrated by three plant objects: $H$. helix, $M$. deliciosa var. borsigiana, S. octophylla. Fungicidal action was detected only in 2 species: E. aureum and S. octophylla.

During indoor introduction, all the studied lianas are rapidly growing and unpretentious. Continuous growth with two or three periods of intense growth (in spring and summer) and moderate growth (in autumn and winter) is typical for $M$. deliciosa var. borsigiana, $E$. aureum, H. helix. Continuous moderate growth during spring and summer, and intense 
growth during autumn and winter was detected for the subtropical species originating from New Zealand, S. octophylla .

The largest number of cases with pronounced activity against bacteria $S$. epidermidis and fungi $C$. albicans was detected during the period of intense growth. During moderate, growth, an increase in the activity against bacteria $E$. coli was observed (Fig. 2).

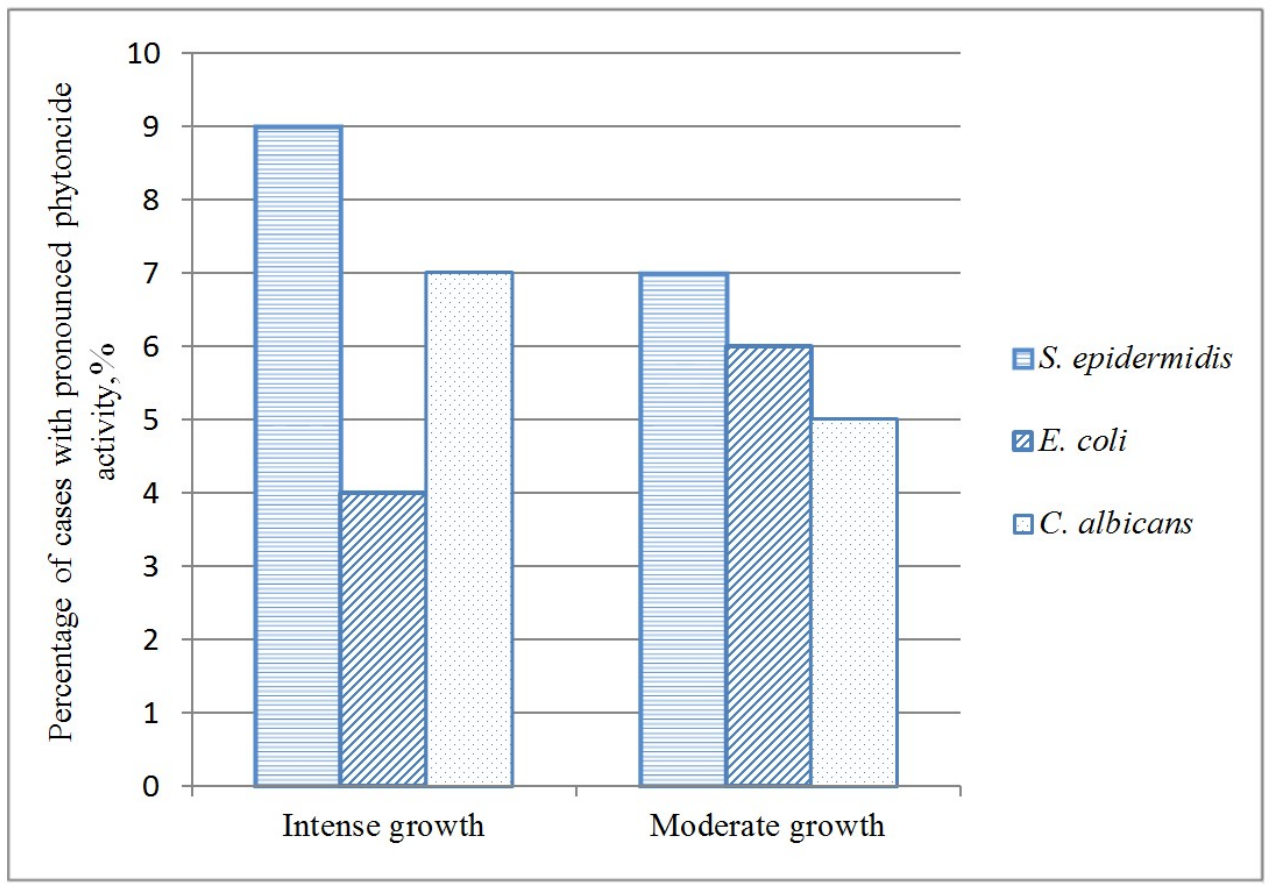

Fig. 2. Number of cases with pronounced antimicrobial activity for plants during different phenological periods.

The maximal bactericidal activity against staphylococcus during intense growth in summer was observed for the following taxons: M. deliciosa var. borsigiana and $S$. octophylla. Fungicidal action was observed for E. aureum and S. octophylla species with the macimal activity during spring-summer and autumn-winter, respectively. Bactericidal effect against $E$. coli was observed for $H$. helix and $M$. deliciosa species with maximal activity during spring-summer and autumn-winter, respectively.

\section{Discussion}

Evaluation of the antimicrobial activity of volatile substances released from the leaves of 3 species and 1 variety of plants from 2 families was carried out for the first time. Species and seasonal specificity of the antimicrobial activity of exometabolites of intact leaves was detected. It was established that all the studied plant taxons demonstrate moderate bactericidal effect against $S$. epidermidis as average over the season.

During intense growth in summer and in autumn-winter period, the highest antifungal and antibacterial (against $S$. epidermidis) activity was observed. During moderate growth in spring and in autumn-winter, an increase in the bactericidal activity against $E$. coli. was detected. Long-term, moderate seasonal activity with maximum in summer was observed for the species originating from New Zealand, S. octophylla, against S. epidermidis. 
Experimental data obtained in the studies provide evidence of species-specific antimicrobial activity against test objects during different periods of seasonal development of plants. The studied lianas may be recommended for wide application in indoor planting for creating favourable environments in aerophytotherapy zones of continuous action.

The work was performed as part of the State Assignment for the Botanical Garden of the Ural Branch of the Russian Academy of Sciences and the State Assignment for Central Siberian Botanical Garden SB RAS. When preparing the publication, the materials of bioresource scientific collection of CSBG SB RAS «Collections of living plants indoors and outdoors», USU 440534 were used.

\section{References}

1 G.N. Ergasheva, Tree Vines in the Dry Subtropics of Tajikistan: Introduction, Biology, Ecology and Use, Dissertation, (Ufa, 2013).

2 V.V. Snezhko, V.V. Krivenko, N.M. Makarchuk, A.K. Hibnev, Fitontsidnye plants in the interiors. Allelopathy in natural and artificial phytocenoses (Collection of scientific papers Naukova Dumka, Kiev, 1982)

3 R. Saswati, D.M. Choudhury, S.B. Paul, IJRAP 4, 15-17 (2013)

4 M. Aman, R.V. Rai, P.V. Samaga, Med. Aromat. Plant. Sci. Biotechnol. 7, 69-72 (2010)

5 V.T. Bakulin, L.N. Chindyaeva, N.V. Tsybulya, Problems of Regional Ecology 6, 6064 (2010)

6 M. Manso, J.P. Castro-Gomes, Renewable and Sustainable Energy Reviews 41, 863$871(2015)$

7 E.M. Elgizawy, Procedia Environ. Sci. Eng. Manag. 34, 119-130 (2016)

8 A. Rahman, F. Yeok, A. Amir, World Acad. Sci. Eng. Technol. 5, 206-214 (2011) 